\title{
Introducing S.A.S.S
}

\author{
Mister Seun Ayoade* \\ Independent Researcher, College of Medicine University of Ibadan, Nigeria.
}

*Corresponding author: Mister Seun Ayoade, Independent Researcher, Physiology, College of Medicine University of Ibadan, Nigeria.

\section{Opinion}

"Any sufficiently advanced technology is indistinguishable from magic"-Arthur C Clarke.

"Mop up operations are what engage most scientists throughout their careers. This paradigm-based research is an attempt to force nature into the pre-formed and relatively inflexible box that the paradigm supplies. No effort is made to call forth new sorts of phenomena, no effort to discover anomalies. When anomalies pop up, they are usually discarded or ignored. Anomalies are usually not even noticed, and no effort is made to invent a new theory-and there is no tolerance for those who try" [1].

SASS is an acronym for Seun Ayoade's Surgical System. It is surgery involving the microzymas [cellular dust] and the Primo Vascular System, surgery based on the Germ Terrain Duality Theory of disease.

The Germ-Terrain duality theory of disease states that the ethology of certain diseases/diseased states is better explained as a complex interplay between germs and the inherent anatomical/ physiological integrity of the body cells.

It argues that the etiology of certain diseases is not fully explained merely by the presence of germs (Germ Theory) or by a mere loss of cellular integrity (Terrain Theory).

As a result, the prevention and treatment of such diseases should focus not just on fighting germs but on maintaining/ restoring the anatomical/physiological cellular integrity.

The Germ-Terrain duality theory is a harmonization of the current Germ Theory (popularized by Louis Pasteur) and the hitherto discarded Terrain Theory (popularized by Pierre Bechamp) [2-4].

SASS and SAMS (Seun Ayoade's Medical System) go hand in hand. SASS applications are numerous.
Picture this: a man is run over by a truck. He is rushed to the hospital and diagnosed with multiple fractures ranging from comminuted to Colles' to Pott's. Twenty-four hours later this same man is sighted playing basketball and tennis, grinning from ear to ear! Magic? A miracle? Neither! This could very well be a reality soon. But only in a future in which scientists and ortho pedists acknowledge the existence of the microzymas and learn to manipulate them.

\section{Accelerated fracture treatment via the manipulation of osteoblastic dust [5] etcetera}

Utilization of Bone mending pills/injections/tablets/syrups/ drips/patches containing nanobots primed to perform bone and muscle surgery at the microzyman and primo vascular/Bonghan corpuscle level [6]. After performing the surgeries the nanobots will be deactivated and expelled in the faeces/urine. The medications will only be triggered if there's a fracture in case of accidental ingestion by toddlers etc.

\section{The bechamp $[\mathrm{B}, \mathrm{b}]$ as unit of microzyman mensuration}

I propose the Bechamp [B, b]as a mensural unit for working with Cellular Dust. "B+" will denote human microzyma/microzyma within a human.

"B-" will denote animal/plant microzyma, while neutral, plain "B" will represent microzyma in the air or on inanimate objects,

Brackets "() []" will denote "power".

The Bechamp, B symbol and the plus or minus signs can be put before or after the value as the scientist prefers. Hence $+B 10(6)$ means a million human microzyma, $10(8) \mathrm{B}$ means a hundred million plant or animal microzymas and B10(9) and 10(9)B represents a billion microzyma taken from the air. 
Lower case "b" will denote microzyma contained in a cubic milli meter of medium, irrespective of number or type of cellular dust. This unit will come in handy in transporting, storing and injecting diverse microzymas.

\section{Agricultural Applications}

The following reads like something out of Harry Potter

"The ground having thus been prepared and having been moistened with water a few kernels of corn were buried in the loose earth the soil was seen to move, and a tiny green blade came slowly to view. This continued to increase in height and size, until in the course of twenty minutes or half an hour of planting (the seed) the stalk or corn was a foot or fifteen inches in height The stalks continued to grow until they were full height and put forth one or more ears of corn"[7].

Whether this event happened or not I do not know. But could similar accelerated crop growth be achieved one day with seeds soaked in liquids impregnated with microzymas-manipulating nanobots? World hunger could be eliminated, and the time and lands required for farming greatly reduced.

Before you laugh, smirk or smugly say to yourself "these things can never be" ask yourself these questions: who would have thought, five hundred years ago, that a man in Russia would softly yet clearly speak to a woman far away in France as if they were sitting on the same sofa through a device called a "phone"?
Who would have thought, just fifty years ago that a dozen movies and a thousand songs would be put on a device as small as a man's finger a "USB", inserted in something called a "flash drive" and played on a "laptop"?

The future is as bright as we can make it!

\section{Acknowledgement}

None

\section{Conflict of intrest}

The author has no conflict of interest.

\section{References}

1. (1962) Structure of Scientific Revolutions.

2. Ayoade S (2017) Etiology, Epidemiology and Therapeutic History of Malaria Validate Germ-Terrain Duality; Postulates Thereof. J Mol Genet Med 11: 261.

3. Ayoade S (2017) Antimalarials Validate the Germ Terrain Duality Theory. JOJ Nurse Health Care 2(5): 555-600

4. Ayoade S (2017) The Differences Between the Germ Theory, the Terrain Theory and the Germ Terrain Duality Theory. JOJ Nurse Health Care 4(2): 555-631.

5. Ayoade S (2018) "A Few More Differences between the Theories Three". ARC Journal of Nursing and Healthcare 4(3): 1-2.

6. Lee BC, Yoon JW, Park SH, Yoon SZ (2013) Toward a theory of the primo vascular system: A hypothetical circulatory system at the subcellular level. Evid Based Complement Alternat Med 2013: 961957.

7. Vine Deloria Jnr. The World We Used to Live In: Remembering the Powers of The Medicine. pp. 27. 